[21] J. Salbeck, M. Schörner, T. Fuhrmann, Thin Solid Films 2002 417, 20.

[22] T. Spehr, R. Pudzich, T. Fuhrmann, J. Salbeck, Org. Electron. 2003, $4,61$.

[23] S. Riechel, U. Lemmer, J. Feldmann, S. Berleb, A. G. Mückl, W. Brütting, A. Gombert, V. Wittwer, Opt. Lett. 2001, 26, 593.

[24] S. Santra, P. Zhang, K. Wang, R. Tapec, W. Tan, Anal. Chem. 2001, $73,4988$.

[25] R. Weigert, A. Colanzi, A. Mironov, R. Buccioni, C. Pericola, M. G. Dciulli, G. Santini, S. Flati, A. Fusella, J. G. Donaldson, M. Di Girolamo, D. Corda, M. A. De Mattteis, A. Luini, J. Biol. Chem. 1997, 272,14200

[26] C. Lombry, C. Bosquillon, V. Preat, R. Vanbever, J. Controlled Release 2002, 83, 331.

[27] S. Haghighat, D. J. Castro, R. B. Lufkin, H. R. Fetterman, J. Soudant, P. H. Ward, R. E. Saxton, Laryngoscope 1992, 102, 81.

[28] D. L. Heintzelman, U. Utzinger, H. Fuchs, A. Zuluaga, K. Gossage, A. M. Gillenwater, R. Jacob, B. Kemp, R. R. Richards-Kortum, Photochem. Photobiol. 2000, 72, 103.

[29] M. Berggren, A. Dodabalapur, R. E. Slusher, A. Timko, O. Nalamasu, Appl. Phys. Lett. 1998, 72, 410.

[30] M. Gaal, C. Gadermaier, H. Plank, E. Moderegger, A. Pogantsch, G. Leising, E. J. W. List, Adv. Mater. 2003, 15, 1165.

[31] J. R. Lawrence, G. A. Turnbull, I. D. W. Samuel, Appl. Phys. Lett. 2003, 82, 4023.

[32] G. Khanarian, and H. Celanese, Opt. Eng. 2001, 40, 1024.

[33] T. Nielsen, D. Nilsson, F. Bundgaard, P. Shi, P. Szabo, O. Geschke, A. Kristensen, J. Vac. Sci. Technol. B 2004, 22, 1770

\section{Bright White Organic Light-Emitting Devices from a Single Active Molecular Material}

\author{
By Marco Mazzeo,* Vincenzo Vitale, Fabio Della Sala, \\ Marco Anni, Giovanna Barbarella, Laura Favaretto, \\ Giovanna Sotgiu, Roberto Cingolani, and \\ Giuseppe Gigli
}

In the last few years, white-light emission from organic compounds has been the subject of increasing interest due to its potential impact on the lighting industry and backlight applications. In order to obtain white light from organic lightemitting devices (OLEDs), the simultaneous excitation of different molecular species emitting at different primary colors is required. ${ }^{[1,2]}$ So far, the most exploited approach has been

[*] Dr. M. Mazzeo, ${ }^{[+]}$Dr. V. Vitale, Dr. F. Della Sala, Dr. M. Anni, Prof. R. Cingolani, Dr. G. Gigli

NNL, National Nanotechnology Laboratory

Istituto Nazionale di Fisica della Materia (INFM)

Dipartimento di Ingegneria dell'Innovazione, Università di Lecce via Arnesano, 1-73100 Lecce (Italy)

E-mail: marco.mazzeo@unile.it

Dr. G. Barbarella, Dr. L. Favaretto, Dr. G. Sotgiu

Consiglio Nazionale delle Ricerche (CNR), ISOF

Area della Ricerca di Bologna, via Gobetti 101, I-40129 Bologna (Italy)

[+] Second address: Dipartimento di Fisica, Università di Lecce, via Arnesano, I-73100 Lecce, Italy. the fabrication of multilayer devices by consecutive evaporations or co-evaporation of different emitting compounds. ${ }^{[1-6]}$ However, this technique requires complex technological processes and a large amount of wasted organic materials, resulting in relatively high fabrication costs. Spin-coating of a blend of different soluble emitters in a single layer seems to be a more cost-effective technique. ${ }^{[7,8]}$ Though cheaper, this approach has the drawback that customized color combinations are not always possible due to Förster transfer from the highenergy emitting material (donor) to the low-energy one (acceptor), which induces emission only from the lower-gap compound.$^{[9,10]}$ An alternative approach which overcomes such a problem is to blend two blue-light-emitting organic molecules of different electron affinities, whose interaction gives rise to exciplex states. ${ }^{[11,12]}$ The combination of the exciplex emission with the blue-light emission of the individual donor molecule results in the generation of white light. However, in both of these approaches the purity of the color emission is strongly dependent on the relative concentration of the different molecular species and, generally, on the applied voltage. This is a problem for lighting applications in which the source intensity (but not the color) has to be varied by changing the applied electrical power. ${ }^{[13]}$

In this frame, the synthesis of a soluble compound showing white-light emission in the solid state is strongly desirable because it would enable the fabrication of a new class of devices which combine the simplicity and low cost of the single-layer spin-coated structures without the problems connected with the material concentration and bias.

In this work, we demonstrate a bright single-layer white OLED realized by spin-coating a single emitting molecular material, namely 3,5-dimethyl-2,6-bis(dimesitylboryl)-dithieno[3,2-b:2', $\left.3^{\prime}-d\right]$ thiophene (compound 1 in Fig. 1). In 1, white electroluminescence is achieved by the superposition of the intrinsic blue-green-light emission (BGE) of the single molecule with a red-shifted emission (RSE) that occurs only in the solid state. The origin of the RSE peak is due to the formation of cross-like dimers between the molecules. This has been demonstrated by optical measurements and theoretical calculations, and also performed on similar compounds functionalized with different substitution patterns (2, 3, and $\mathbf{4}$ in Fig. 1) in order to control the self-assembling of the molecules. By virtue of the excellent properties of compound $\mathbf{1}$, namely the good electron-acceptor characteristics of the dimesitylboryl moieties, and the peculiar morphology of the spin-coated films, it was possible to obtain white-light electroluminescence (EL) with a maximum luminance of $3800 \mathrm{~cd} \mathrm{~m}^{-2}$ at $18 \mathrm{~V}$ and an external quantum efficiency (QE) of $0.35 \%$, paving the way to the fabrication of low-cost single-active-material white-light-emitting devices.

In Figure 2 we show the photoluminescence (PL) and absorption spectra of $\mathbf{1}$ in solution (Fig. 2a) and in the solid state (Fig. 2b). The absorption spectrum in the solid state does not show significant differences with respect to that in solution. Instead, strong changes are observed in the emission spectra. In solution, only the BGE is observed, whereas in solid-state 

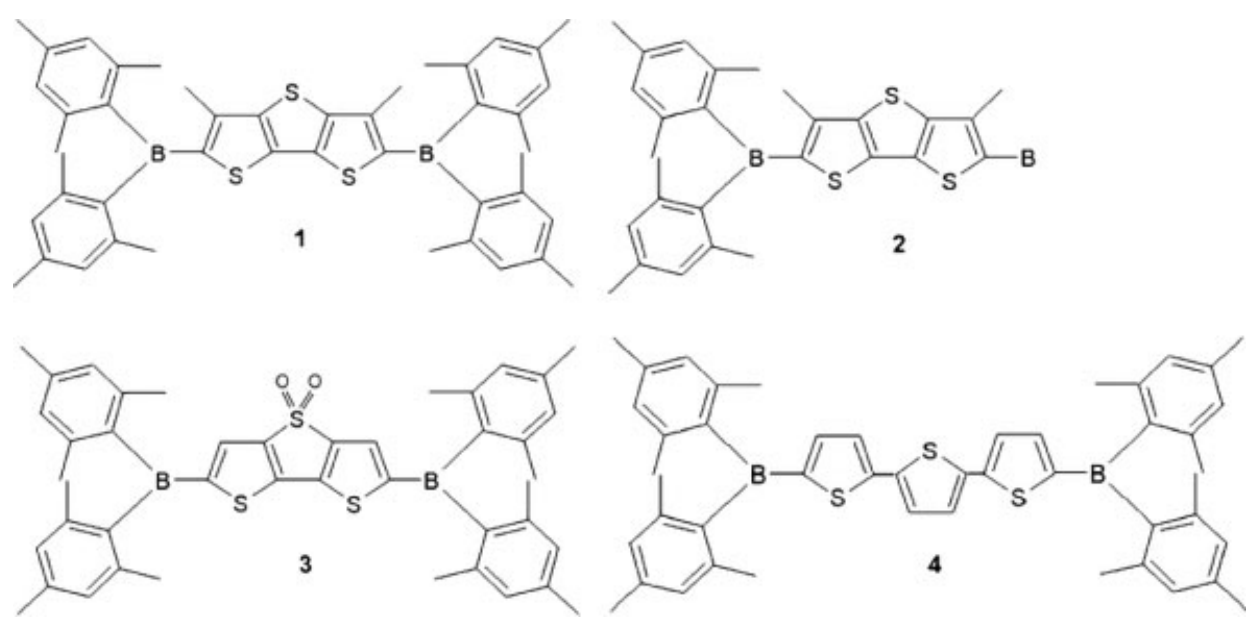

Figure 1. Molecular structures of compounds 1-4.

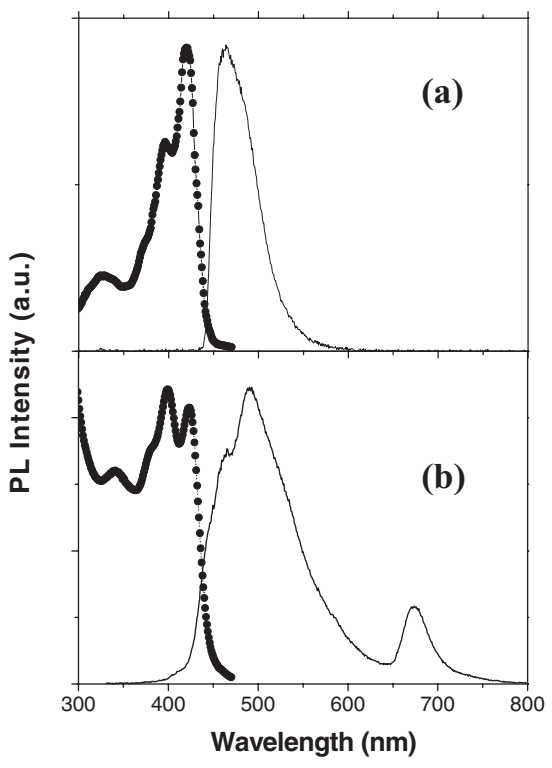

Figure 2. PL (solid line) and absorption spectra (dotted line) at room temperature of $\mathbf{1}$ a) in tetrahydrofuran (THF) solution and b) in the solid state.

films the additional narrow RSE at $680 \mathrm{~nm}(1.82 \mathrm{eV})$ appears. We underline that this low-energy peak is absent in solution regardless of the concentration range $\left(10^{-5}-10^{-2} \mathrm{M}\right)$, indicating that the RSE is peculiar to the solid state. Similar RSEs in the solid state have been observed in various organic materials, and could be assigned to triplets activated in the solid state or particular aggregation states. ${ }^{[14-16]}$

In order to check this point, we investigated the dynamical processes involved in the PL emission of solid-state films by time-resolved photoluminescence (TR-PL). The decay dynamics of the BGE at $480 \mathrm{~nm}$ and of the RSE at $680 \mathrm{~nm}$ are shown in Figure 3. The PL decay at $480 \mathrm{~nm}$ is bi-exponential, with a fast decay time of $30 \pm 1$ ps and a slow decay time of $91 \pm 1$ ps. The slow decay time is comparable to that of the

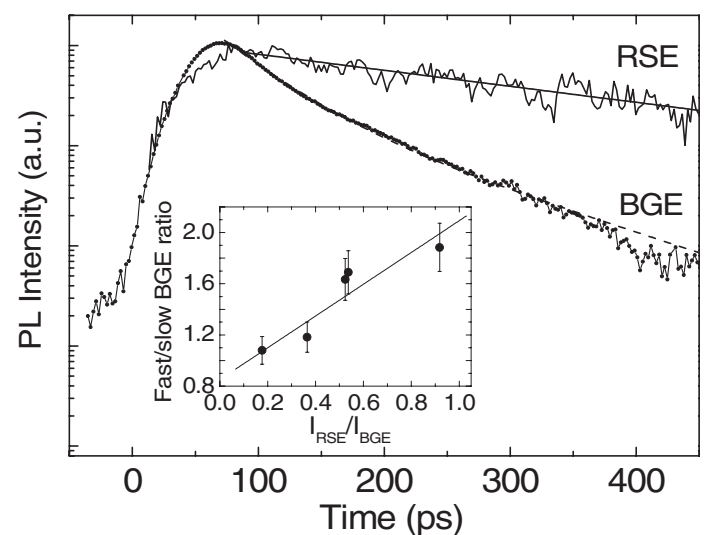

Figure 3. Decay dynamics of the BGE emission at $480 \mathrm{~nm}$ (dotted line) and of the RSE at $680 \mathrm{~nm}$ (solid line) in the solid state of 1. Inset: Ratio of the amplitudes of the fast/slow components of the BGE relaxation versus the RSE/BGE intensity. These data have been obtained by TR-PL measurements by exciting regions of the films close to the edges. At the edges of spin-coated films, the sample morphology is, in fact, generally less homogenous, and different aggregate concentrations can be more easily detected.

molecule in THF solution (not shown). The fast decay process is instead related to the formation of the RSE, as demonstrated by the linear dependence of the ratio between the amplitudes of the fast and slow components of the BGE relaxation on the RSE/BGE intensity (inset of Fig. 3). The PL signal at $680 \mathrm{~nm}$ shows a slower decay time of about $1700 \pm 170 \mathrm{ps}$. Such a decay time allows us to exclude that the RSE could be due to triplet emission, which usually shows much longer decay times, ${ }^{[16]}$ and suggests that it is likely due to aggregates or excimers.

However, aggregates and excimer states are generally characterized by broad PL spectra, ${ }^{[14-16]}$ whereas a narrow linewidth is observed for the RSE. This is very likely due to the particular supramolecular arrangements which compound $\mathbf{1}$ can assume in the aggregate state. Due to the planar and rigid 
conformation of the inner core and the presence of the large mesityl substituents, molecules of $\mathbf{1}$ are likely to fit together in a cross-like configuration as shown in Figure 4a, with very small movements through translations of the molecules along the $x-y$ axis or small angular $(\alpha)$ deviations being allowed. Commonly observed ${ }^{[16]} \mathrm{H}$-type dimeric aggregates (i.e., with

(a)
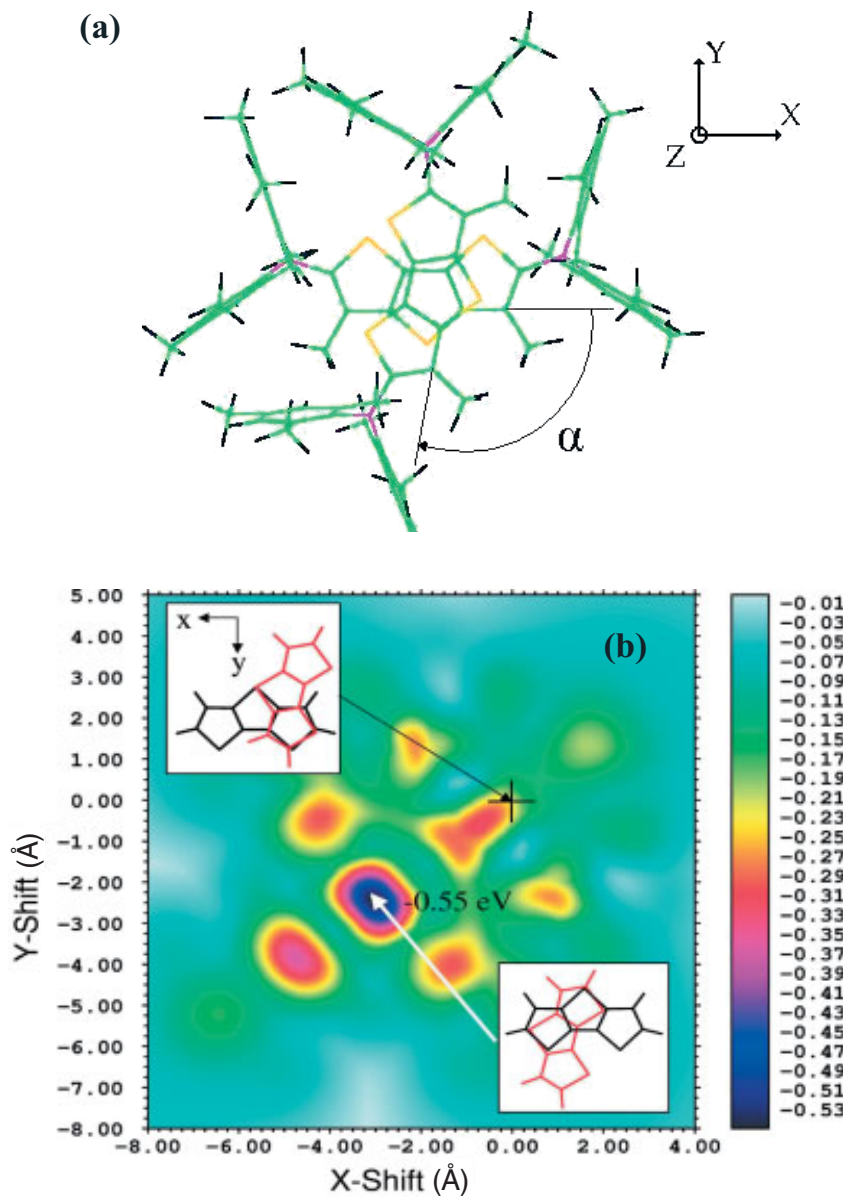

Figure 4. a) Molecular structure of two interacting molecules forming a cross-like dimer. b) Intermediate neglect of differential overlap/single configuration interaction (INDO/SCI) excitation-energy shifts due to intermolecular interactions. The scale on the right corresponds to calculated excitation energy shifts.

$\alpha=180^{\circ}$ ) are, in contrast, completely forbidden for compound $\mathbf{1}$, due to the repulsion of the mesityl substituents. In such fixed cross-like configurations, the RSE broadening induced by supramolecular conformation dispersion is thus strongly reduced. The broader linewidth of the single-molecule emission (i.e., BGE), in comparison to that of the RSE, is due to disorder, solid-state effects, and the presence of vibrational replicas.

In order to check the viability of this hypothesis, and in particular, to verify whether the cross-like conformation is consistent with the measured $0.7 \mathrm{eV}$ shift of the RSE peak with respect the BGE peak in the solid-state, accurate quantum mechanical calculations have been carried out. We calculated the excitation energies of various dimer conformations of the unsubstituted dithienothiophene (DTT) using the intermediate neglect of differential overlap/single configuration interaction (INDO/SCI) approach. ${ }^{[17]}$ The mesityl and methyl substituents have been neglected because they only induce steric effects, and thus have minor influences on the intermolecularinteraction effects on excitation energy. We ran a batch of simulations, varying the coordinates $(x, y)$, the angle $\alpha$, and the distance between molecular planes. In Figure $4 b$, we report the excitation-energy differences between the single DTT molecule and the dimers for all the $(x, y)$ positions with $\alpha$ fixed at $102^{\circ}$ (for which the minimum energy is obtained) and the distance between molecular planes fixed at $3.2 \AA$ (the distance of the molecular dimers in the excited state, which is smaller than that in the ground state ${ }^{[16]}$ ). The dimer for which the two central sulfur atoms overlap represents the center coordinate; then, one molecule (the black one in Fig. 4b) is fixed, whereas the other one (the red one) is shifted (upperleft inset in Fig. 4b). We note that the potential-energy surface (PES) of real dimers will be dominated by the repulsion of all the dimesitylboryl substituents, so that only the central region of Figure $4 \mathrm{~b}$ represents attainable configurations. For this reason, the computation of the PES is computationally prohibitive. Figure $4 b$ shows that, for the configuration depicted in the lower-right inset, intermolecular interactions induce a redshift as high as $0.55 \mathrm{eV}$. This calculated value is in good agreement with the experimental result $(0.7 \mathrm{eV})$, considering the approximations that were employed. We note that, in such cross-like configurations, the molecular-transition-dipole moments, aligned with the principal axis of the molecule, are almost orthogonal to each other. Thus, in the dipole-dipole approximation of the exciton-resonance interaction, no intermolecular effects on the excitation energies are present. ${ }^{[16]}$ A localized-configuration interaction analysis ${ }^{[18]}$ shows that $40 \%$ of the excited-state dimer wavefunction is composed of charge-transfer contributions.

The main result from the plot in Figure $4 \mathrm{~b}$ is that the excitation-energy shift is dominated by only one deep minimum. This means that only one conformation would be responsible for the RSE, in agreement with the narrow red-shifted emission peak observed in the solid state.

Compound $\mathbf{1}$ has been used as an active material in lightemitting diodes by spin-coating the emissive layer between indium tin oxide/poly(ethylenedioxythiophene):poly(styrene sulfonate) (ITO/PEDOT:PSS) and LiF/Al layers (see Experimental), used as the anode and cathode, respectively. PEDOT:PSS and LiF layers were used in order to enhance the carrier injection into the emissive layer, as demonstrated by electro-absorption spectroscopy measurements. ${ }^{[19,20]}$ The EL spectrum is reported in Figure 5. The emissive features are quite similar to the PL spectrum obtained in the solid state, however, the low-energy peak at $680 \mathrm{~nm}$ is more intense than in the PL spectrum, indicating that a more efficient population of the aggregation state occurs upon electrical injection. This effect results in a clear white-light emission with color co- 


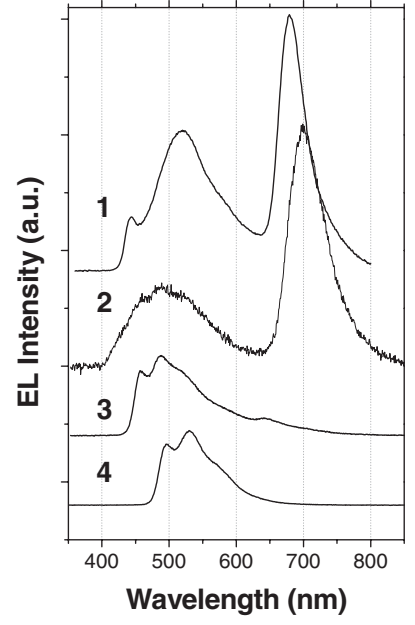

Figure 5. EL spectra of compounds 1-4.

ordinates $(0.31,0.42)$, according to the Commission Internationale de L'Eclairage (CIE) (Fig. 6a). The color coordinates exhibit variations of less than $7 \%$, showing a very high stability at the applied voltage. The efficient charge injection from the $\mathrm{LiF} / \mathrm{Al}$ contact into the active material and the excellent
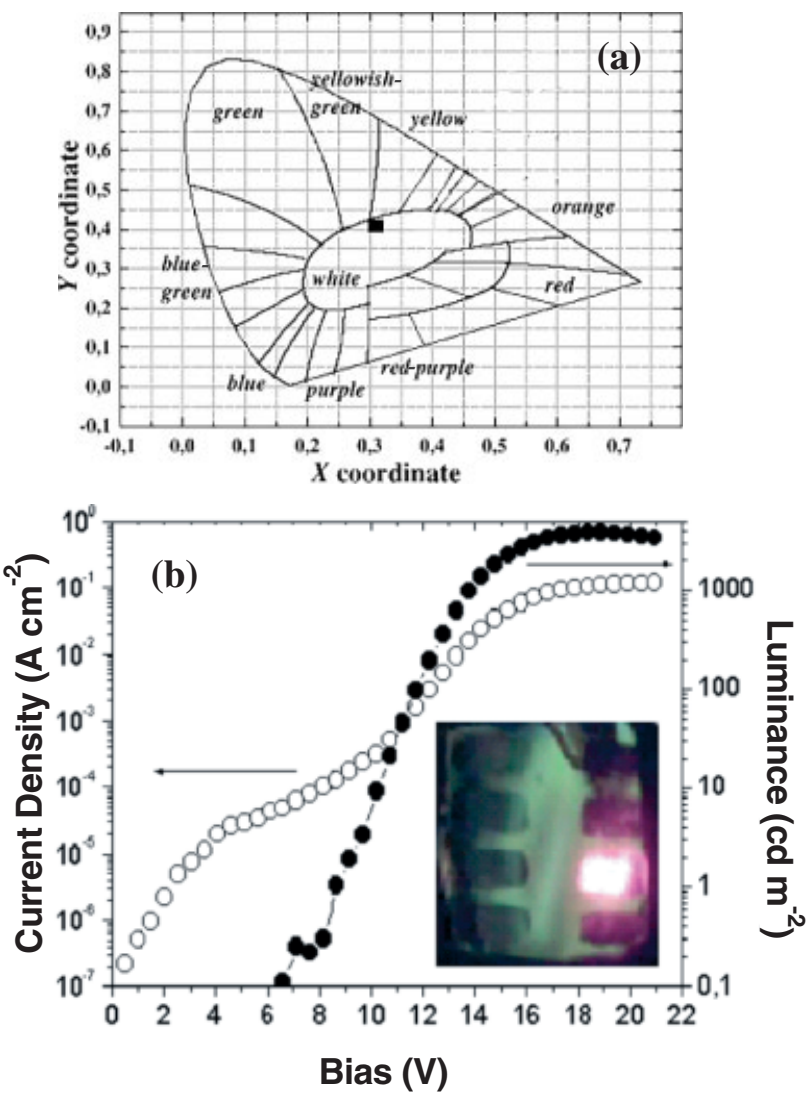

Figure 6. a) $\mathrm{CIE}$ coordinates of the $\mathrm{EL}$ emission. b) Luminance and current density versus the applied voltage of the ITO/PEDOT:PSS/1/LiF/AI device. Inset: photo of a working OLED. electron-accepting properties of the molecule ${ }^{[19-21]}$ allowed us to obtain no-bias-dependent EL with luminance up to $3800 \mathrm{~cd} \mathrm{~m}^{-2}$ and a quantum efficiency of $0.35 \%$ (Fig. 6b), in air, comparable to the single-layer devices realized by spincoating different primary-color-light emitters. The device also shows highly stable color emission over time. We emphasize that, to our knowledge, this is the first white OLED with emission from a single active molecular compound.

In order to corroborate this model and to better clarify the effects of the supramolecular packing on the optical properties, we synthesized a series of molecules having structures similar to 1 but with different functionalization patterns. The analyzed molecules are displayed in Figure 1, and consist of an asymmetric 3,5-dimethyl-2-dimesitylboryl-dithieno[3,2$\left.b: 2^{\prime}, 3^{\prime}-d\right]$ thiophene in which only one dimesitylboryl moiety is grafted to the central core (2), a 2,6-bis(dimesitylboryl)dithieno[3,2-b:2, $\left.3^{\prime}-d\right]$ thiophene-4,4-dioxide in which the central thienyl sulfur atom is substituted with two oxygen atoms

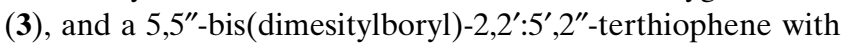
a flexible central core (4) $\cdot{ }^{[21]}$ Compounds $2-4$ have been used as active materials in OLEDs with the same device structure used for $\mathbf{1}$ above.

In compound 2, one of the two large lateral dimesitylboryl groups has been removed in order to reduce the effects of the steric hindrance. That is, by reducing the repulsion of the substituents, the constraint of the molecules to assume only a well-defined cross-like dimer conformation is removed, and shortening the intermolecular distances between the molecules in the solid state. Devices with compound $\mathbf{2}$ as an active material show poor brightness levels and high turn-on biases $(25 \mathrm{~V})$, probably because of the lower number of boron atoms (electron acceptors) and consequently, the poor balancing between opposite charges. More importantly, due to the smaller distance between the two molecules forming the cross-like dimer, the EL of this compound shows an even more red-shifted peak (at $700 \mathrm{~nm}$ ) than that of compound $\mathbf{1}$ (Fig. 5), consistent with our model. The linewidth of the redshifted peak is larger in compound $\mathbf{2}$ (full width at half maximum FWHM $=159 \mathrm{meV})$ than in $\mathbf{1}(\mathrm{FWHM}=114 \mathrm{meV})$, as expected from the lowering of the conformational potential energy barrier in compound $\mathbf{2}$, because of the lack of one lateral dimesitylboryl group.

In contrast to $\mathbf{1}$ and $\mathbf{2}$, compounds $\mathbf{3}$ and $\mathbf{4}$ do not show any red-shifted additional peak in the EL spectra. This is in agreement with our model. In fact, in compound $\mathbf{3}$ the two oxygen atoms are arranged in a plane perpendicular to the plane of the rigid core, ${ }^{[22]}$ thus moving the molecules apart and preventing dimer formation. Finally, in $\mathbf{4}$, in which the rigid core is replaced by a conformationally flexible terthiophene, the formation of the cross-like dimer is not favored due to the rotational degrees of freedom of the terthiophene core. ${ }^{[23]}$

In conclusion, we have presented a new class of bright single-layer spin-coated OLEDs emitting white light from a single active oligothiophene-based compound in which proper functionalization allows one to control the supramolecular organization in the solid state and the formation of new inter- 
molecular emitting species. White-light emission is achieved by the superposition of the broad blue-green-light emission originating from the single molecule and a red-shifted narrow peak which we assign to the formation of cross-like dimers in the solid state. This hypothesis is proposed and discussed in terms of theoretical calculations and various experiments. The realized devices show bright luminance, up to $3800 \mathrm{~cd} \mathrm{~m}^{-2}$ and a QE of $0.35 \%$ in air, comparable to those reported in the literature for spin-coated blends. Fine-tuning of the emission can, in principle, be obtained by exploring other functionalization patterns to better control the degree of aggregation. The simplicity of the device structure, together with the use of a single molecular specie which avoids material-concentrationand voltage-dependent drawbacks, makes our white-lightemitting devices very promising for lighting applications.

\section{Experimental}

General Procedures: Organic solvents were dried following standard procedures. Analytical thin-layer chromatography (TLC) was carried out using $0.2 \mathrm{~mm}$ sheets of silica gel $60 \mathrm{~F}_{254}$ and $0.2 \mathrm{~mm}$ sheets of neutral aluminum oxide $60 \mathrm{~F}_{254}$. Butyl lithium (BuLi) $(2.5 \mathrm{M}$ in hexane) and dimesitylboron fluoride were commercially available. Compound 4 was prepared as described in the literature [20]. ${ }^{1} \mathrm{H}$ and ${ }^{13} \mathrm{C}$ NMR spectra were obtained with a Varian Mercury 400 spectrometer.

2,6-Bis (dimesitylboryl)-3,5-dimethyl-dithieno[3,2-b:2',3'-d/thiophene (1) and 2-dimesitylboryl-3,5-dimethyl-dithieno [3,2-b:2',3'-d]thiophene

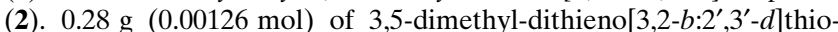
phene [22] was first dissolved in $20 \mathrm{~mL}$ of anhydrous tetrahydrofuran (THF), then $1.12 \mathrm{~mL}(0.0028 \mathrm{~mol}) \mathrm{BuLi}(2.5 \mathrm{M}$ in hexane) was added dropwise. After $4 \mathrm{~h}, 0.83 \mathrm{~g}(0.0028 \mathrm{~mol})$ of dimesitylboron fluoride $(90 \%)$, dissolved in $10 \mathrm{~mL}$ anhydrous THF, was added and the mixture stirred overnight at room temperature (RT). Afterwards, $20 \mathrm{~mL}$ of deionized water was added and the organic layer separated, dried with $\mathrm{Na}_{2} \mathrm{SO}_{4}$, and evaporated. The obtained brown solid was chromatographed on silica gel using petroleum ether (30:40)/pentane/cyclohexane in a 40:30:30 ratio. The first fraction obtained, compound $\mathbf{2}$, consisted of a light-yellow solid $(0.09 \mathrm{~g}, 15 \%$ yield $)$. MS m/e 472 $\left(M^{\bullet+}\right)$; mp $228^{\circ} \mathrm{C}$; UV $\left(\mathrm{CH}_{2} \mathrm{Cl}_{2}\right) \lambda_{\max }: 382 \mathrm{~nm} .{ }^{1} \mathrm{H}$ NMR $\left(\mathrm{CDCl}_{3}\right.$, TMS, $\delta): 7.04\left(\mathrm{q},{ }^{4} J=1.0 \mathrm{~Hz}, 1 \mathrm{H}\right), 6.82(\mathrm{~m}, 4 \mathrm{H}), 2.37\left(\mathrm{~d},{ }^{4} J=1.0 \mathrm{~Hz}\right.$, $3 \mathrm{H}), 2.30$ (broad s, 6H), 2.14 (broad s, $15 \mathrm{H}) \cdot{ }^{13} \mathrm{C} \mathrm{NMR}\left(\mathrm{CDCl}_{3}, \mathrm{TMS}\right.$, $\delta$ ): 146.1, 144.6, 141.8, 140.2, 138.7, 131.1, 130.5, 128.3 (broad), 123.3, 23.0 (broad), 21.3, 15.0, 14.6. The second fraction, compound $\mathbf{1}$, consisted of $0.36 \mathrm{~g}$ ( $43 \%$ yield) of a light-yellow solid. MS $m / e 720\left(\mathrm{M}^{\bullet+}\right)$; $\mathrm{mp} 328-330^{\circ} \mathrm{C}$; UV $\left(\mathrm{CH}_{2} \mathrm{Cl}_{2}\right) \lambda_{\text {max }}: 420 \mathrm{~nm} .{ }^{1} \mathrm{H}$ NMR $\left(\mathrm{CDCl}_{3}, \mathrm{TMS}\right.$, $\delta): 6.80(\mathrm{~s}, 8 \mathrm{H}), 2.30(\mathrm{~s}, 12 \mathrm{H}), 2.12(\mathrm{~s}, 30 \mathrm{H}) .{ }^{13} \mathrm{C} \mathrm{NMR}\left(\mathrm{CDCl}_{3}\right.$, TMS, $\delta): 148.5,147.8,141.5,140.7$ (broad), 139.5, 138.9, 128.2, 23.0, 21.3, 14.9.

2,6-Bis (dimesitylboryl)-dithieno[3,2-b:2',3'-d $]$ thiophene-4,4-dioxide (3). To a solution of $0.06 \mathrm{~g}(0.00026 \mathrm{~mol})$ of dithieno[3,2-b:2', $\left.3^{\prime}-d\right]$ thiophene dioxide in $4 \mathrm{~mL}$ anhydrous THF, kept at $-20^{\circ} \mathrm{C}$, was added dropwise $0.23 \mathrm{~mL}(0.00057 \mathrm{~mol}) \mathrm{BuLi} 2.5 \mathrm{M}$ in hexane. After $3 \mathrm{~h}$, the mixture was added dropwise to a solution of $0.18 \mathrm{~g}(0.0006 \mathrm{~mol})$ of dimesitylboron fluoride $(90 \%)$ in $3.5 \mathrm{~mL}$ anhydrous ethyl ether kept at $-60{ }^{\circ} \mathrm{C}$. The mixture was stirred overnight at RT, then $5 \mathrm{~mL}$ of deionized water were added, the organic phase separated, dried with $\mathrm{Na}_{2} \mathrm{SO}_{4}$, and evaporated. The solid obtained was chromatographed on silica gel using pentane/methylene chloride/ethyl acetate in a ratio of 90:5:5. A light-yellow solid was obtained $(0.03 \mathrm{~g}, 16 \%$ yield $)$. MS m/e $724\left(M^{\bullet+}\right)$; mp $200{ }^{\circ} \mathrm{C}$; UV $\left(\mathrm{CH}_{2} \mathrm{Cl}_{2}\right) \delta_{\max }: 432 \mathrm{~nm} .{ }^{1} \mathrm{H}$ NMR $\left(\mathrm{CDCl}_{3}, \mathrm{TMS}, \delta\right): 7.46(\mathrm{~s}, 2 \mathrm{H}), 6.84(\mathrm{~s}, 8 \mathrm{H}), 2.31(\mathrm{~s}, 12 \mathrm{H}), 2.12(\mathrm{~s}, 24 \mathrm{H})$. ${ }^{13} \mathrm{C}$ NMR (CDCl 3 , TMS, $\delta$ ): 146.5, 145.8, 140.9, 139.9 (broad), 139.7, 131.1, 128.5, 23.5, 21.2.
Computational Details: Calculations have been done using the inhouse-developed Molecular Orbital Configuration Interaction (MOCI) program.

Standard intermediate neglect of differential overlap/single configuration interaction (INDO/SCI) parameters have been used. Twelve occupied orbitals and ten virtual orbitals have been included in the configuration interaction space; doubling the number of occupied and virtual orbitals has been found to cause a negligible $(0.05 \mathrm{eV})$ reduction of the lowest-excited-state energy. Neutral ground-state geometries have been used; geometry-relaxation effects on the single molecules have been found to have negligible effects on the values and on the shape of Figure 4b.

Optical Measurements: Photoluminescence (PL) measurements were performed on thin films, cast from THF solution $\left(10^{-2} \mathrm{M}\right)$, by exciting the samples with an $\mathrm{He}-\mathrm{Cd}$ laser $(325 \mathrm{~nm})$ and collecting the signal with a charge-coupled device (CCD) spectrograph (Ocean Optics). Time-resolved PL measurements were made using the second harmonic $(\lambda=390 \mathrm{~nm})$ of a Ti-sapphire mode-locked laser delivering 2 ps pulses at a repetition rate of $82 \mathrm{MHz}$. The luminescence was dispersed by a $0.24 \mathrm{~m}$ single monochromator coupled with a streak camera equipped with a two-dimensional CCD. The overall time resolution was about 8 ps.

Device: The organic light-emitting devices were prepared by spincoating a hole-transporting layer, namely poly(3,4-ethylenedioxythiophene) (PEDOT) doped with poly(styrene sulfonate) (PSS) onto $\mathrm{O}_{2-}$ plasma-treated indium tin oxide (ITO)-coated glass substrates. Subsequently, we spin-cast the active medium (e.g., 1), and finally, a LiF layer $(4.5 \mathrm{~nm})$ followed by an $\mathrm{Al}$ electrode $(70 \mathrm{~nm})$ were deposited by thermal evaporation $\left(10^{-6} \mathrm{mbar}\right)$. The device characterizations were performed at RT in air.

Received: May 3, 2004 Final version: September 14, 2004

[1] G. Li, J. Shinar, Appl. Phys. Lett. 2003, 83, 5359.

[2] R. S. Deshpande, V. Bulovic, S. R. Forrest, Appl. Phys. Lett. 1999, 75,888

[3] C. H. Kim, J. Shinar, Appl. Phys. Lett. 2002, 80, 2201.

[4] Y. S. Huang, J. H. Jou, W. K. Weng, J. M. Liu, Appl. Phys. Lett. 2002, 80, 2782.

[5] K. O. Cheon, J. Shinar, Appl. Phys. Lett. 2002, 81, 1738.

[6] C. H. Chuen, Y. T. Tao, Appl. Phys. Lett. 2002, 81, 4499.

[7] M. Granstrom, O. Inganas, Appl. Phys. Lett. 1996, 68, 147

[8] S. Tasch, E. J. W. List, O. Ekström, W. Graupner, G. Leising, P. Schlichting, U. Rohr, Y. Geerts, U. Scherf, K. Müllen, Appl. Phys. Lett. 1997, 71, 2883 .

[9] T. Forster, Discuss. Faraday Soc. 1959, $27,7$.

[10] M. Anni, G. Gigli, V. Paladini, R. Cingolani, G. Barbarella, L. Favaretto, G. Sotgiu, M. Zambianchi, Appl. Phys. Lett. 2000, 77, 2458.

[11] J. Thompson, R. I. R. Blyth, M. Mazzeo, M. Anni, G. Gigli, R. Cingolani, Appl. Phys. Lett. 2001, 79, 560.

[12] M. Mazzeo, D. Pisignano, F. Della Sala, J. Thompson, R. I. R. Blyth, G. Gigli, R. Cingolani, L. Favaretto, G. Barbarella, Appl. Phys. Lett. 2003, 82, 334.

[13] In particular, even if in the blend, this problem can be limited by properly designing the molecules; a critical dependence on the deposition and external conditions is, in general, always present. Al these factors make accurate control of all the deposition and materials parameters necessary, thus resulting in an increase of the production costs.

[14] J. M. Lupton, M. R. Craig, E. W. Meijer, Appl. Phys. Lett. 2002, 80 , 4489.

[15] W. T. Yip, D. H. Levy, J. Phys. Chem. 1996, 100, 11539.

[16] M. Pope, C. E. Swenberg, Electronic Processes in Organic Crystals, Clarendon, Oxford, UK 1982.

[17] J. E. Ridley, M. C. Zerner, Theor. Chim. Acta 1973, 32, 111. 
[18] R. Paolesse, L. Jaquinod, F. Della Sala, D. J. Nurco, L. Prodi, M. Montalti, C. Di Natale, A. D’Amico, A. Di Carlo, P. Lugli, K. Smith, J. Am. Chem. Soc. 2000, 122, 11295.

[19] T. M. Brown, J. S. Kim, R. H. Friend, F. Cacialli, R. Daik, W. J. Feast, Appl. Phys. Lett. 1999, 75, 1679.

[20] T. M. Brown, R. H. Friend, I. S. Millard, D. J. Lacey, J. H. Burroughes, F. Cacialli, Appl. Phys. Lett. 2001, 79, 174.

[21] T. Noda, H. Ogawa, Y. Shirota, J. Am. Chem. Soc. 1998, 120, 9714

[22] G. Barbarella, L. Favaretto, G. Sotgiu, L. Antolini, G. Gigli, R. Cingolani, A. Bongini, Chem. Mater. 2001, 13, 4112.

[23] Moreover, we also stress that the red-shifted emission could be not due to defects and/or impurities, because all the investigated compounds have been synthesized and purified with the same procedure, but the low energy peaks are present only in compounds $\mathbf{1}$ and $\mathbf{2}$, where they are expected.

\section{Photolithographic Patterning of Ring-Opening Metathesis Catalysts on Silicon**}

\author{
By Robert F. Harris, Melissa J. Ricci, \\ Richard A. Farrer, Julie Praino, Scott J. Miller, \\ Bahaa E. A. Saleh, Malvin C. Teich, and \\ John T. Fourkas*
}

The patterning of polymers on surfaces for use in applications such as organic electronic systems, ${ }^{[1]}$ optical devices, ${ }^{[2]}$ photoresist technology, ${ }^{[3,4]}$ and biological sensors ${ }^{[5]}$ has attracted increasing attention in recent years. Surface-bound polymers can be created by a variety of methods, including radical, anionic, and cationic polymerization, ${ }^{[6-9]}$ scannedprobe lithography, ${ }^{[10]}$ inkjet $^{[11]}$ and screen printing, ${ }^{[12]}$ spincoating, ${ }^{[13]}$ and self-assembled monolayer formation via micro-contact printing $(\mu \mathrm{CP}) \cdot{ }^{[14-16]}$ In addition, it has been demonstrated $^{[4,17-19]}$ that patterned, surface-bound polymers can be grown on surfaces using $\mu \mathrm{CP}$ in conjunction with ringopening metathesis polymerization (ROMP). One limitation of the current methodologies for the patterning of surfacebound polymers is the lack of compatibility with conventional photolithographic technologies. A photolithographic ROMPbased method for creating patterned polymers would facilitate the creation of non-silicon-based structures using proto-

[*] Prof. J. T. Fourkas, Dr. R. F. Harris,

M. J. Ricci, Dr. R. A. Farrer, Prof. S. J. Miller

Eugene F. Merkert Chemistry Center

Boston College, Chestnut Hill, MA 02467-3860 (USA)

E-mail: fourkas@bc.edu

J. Praino, Prof. B. E. A. Saleh, Prof. M. C. Teich

Department of Electrical and Computer Engineering

Boston University, Boston, MA 02215 (USA)

[*** This work was supported by the National Science Foundation (Grant ECS-0088438). J. T. F is a Research Corporation Cottrell Scholar and a Camille Dreyfus Teacher-Scholar. cols that are consistent with those employed in the fabrication of silicon-based structures. In this report, we demonstrate the photolithographic patterning of ROMP catalysts on oxide layers above $\mathrm{Si}(100)$ surfaces and the subsequent growth of both linear and highly crosslinked polymers on these surfaces.

Recent reports have provided insights into the thermolytic decomposition pathways of typical ruthenium carbene complexes used in olefin metathesis ${ }^{[20]}$ and have elucidated the mechanism and activity of this class of catalyst. ${ }^{[21]}$ This work pointed to the dissociation of a phosphine ligand as a critical step in both the activity of the catalyst and its lifetime. It has also been demonstrated ${ }^{[22]}$ that exposure of the ruthenium catalyst 2 to UV light causes a photochemical release of the phosphine ligand. The release of the ligand in the presence of a suitable monomer results in an increase of catalyst activity. We hypothesized that if photo-induced phosphine release was carried out in the absence of a monomer, side reactions could render the catalyst inactive. We verified this hypothesis by dissolving catalyst $\mathbf{2}$ in methylene chloride, exposing the solution to UV light for varying time intervals, and then introducing norbornene monomer. The degree of polymerization decreased with increased exposure time, and with sufficient exposure time polymerization could be eliminated completely. We also found that if the inactivation process was performed under an inert atmosphere, the catalyst remained competent even after prolonged exposure to UV light. While the exact mechanism of light-initiated catalyst decomposition is unknown, ${ }^{1} \mathrm{H}$ NMR analysis of post-exposure catalyst solutions indicates the presence of benzaldehyde. It is unlikely that photodeactivation of the catalysts proceeds via the same bimolecular mechanism ${ }^{[20,21]}$ observed for deactivation in the absence of irradiation, particularly on surfaces. Ongoing studies should provide more insight into the exact mechanism of inactivation.

Having demonstrated the photodeactivation of ROMP catalysts, we sought to use this technique to pattern polymers on surfaces via the scheme in Figure 1a. Si(100) wafers $(1 \mathrm{~cm} \times 1 \mathrm{~cm})$ with a $10000 \AA$ oxide layer were functionalized with trichloro(5-norbornen-2-yl)silane $\mathbf{1}$ and then exposed to a ruthenium metathesis catalyst in order to bind the catalyst to the surface. Catalyst inactivation was effected by exposing the entire surface of the wafer to UV light for $45 \mathrm{~min}$. The wafers were then placed in a solution of norbornene 6. As expected, there was no appreciable polymer growth on exposed wafers when compared to unexposed control wafers. This methodology proved successful with all surface-bound variants of Grubbs' ruthenium-based metathesis catalysts that we investigated: 2 , $\mathrm{RuCl}_{2}\left(\mathrm{PCy}_{3}\right)_{2}(=\mathrm{CHPh}),{ }^{[23]} \quad \mathbf{3}, \quad \mathrm{RuCl}_{2}\left(\mathrm{H}_{2} \mathrm{IMES}\right)\left(\mathrm{PCy}_{3}\right)$ $(=\mathrm{CHPh}),{ }^{[24]}$ 4, $\mathrm{RuCl}_{2}\left(\mathrm{H}_{2} \mathrm{IMES}\right)(\mathrm{Py})_{2}(=\mathrm{CHPh}),{ }^{[25]}$ and 5, $\mathrm{RuCl}_{2}\left(\mathrm{H}_{2} \mathrm{IMES}\right)(m-\mathrm{BrPy})_{2}(=\mathrm{CHPh})(\mathbf{5})^{[26]}$ (Fig. 1b).

Having established that surface-immobilized ruthenium metathesis catalysts can be inactivated using light, the possibility of selective inactivation using a simple photolithographic technique was investigated. A wafer with ruthenium catalyst bound to the surface was placed in direct contact with an optical test mask (Fig. 2a). The mask and wafer were then 